\title{
ABSTRACTS
}

\section{I-DETACHMENT OF THE RETINA}

Gonin, J. (Lausanne).-The pathogenesis and pathological anatomy of retinal detachments, excluding those due to trauma, to neoplasms, and to parasites. (Pathogénie et anatomie pathologique des décollements retiniens, à l'exclusion des décollements traumatiques, néoplastiques et parasitaires.) "Report presented to La Société française d'Ophtal."

This report, by an acknowledged authority on the subject of detachment of the retina, is of sufficient value and interest to deserve a somewhat lengthy abstract.

By way of a short designation for the group of cases embraced by his title, Gonin suggests the name " idiopathic," the common characteristics of the cases being : that they present no apparent participation of the other ocular membranes, that they are free from external irritation, that most of them have no known cause, and that, as a rule, there are no prodromata, or these are slight and consist of floating spots or luminous phenomena which make their appearance a few days or hours before the occurrence of the detachment. The frequency of these cases is shown by the author's figures. His material consists of 200 traumatic cases, 50 due to the presence of a neoplasm and only 37 " idiopathic," of which one half are useless for detailed examination.

Clinical features. The onset may be quite sudden, and associated with cough, effort while stooping, the lifting of a heavy weight or the congestion produced by a hot bath. In these cases usually the upper part of the fundus is the one affected. In others the onset is rapid, but less sudden, announced by some ill-defined sensation, and causing the patient little uneasiness unless there is metamorphopsia due to involvement of the macula. In a third group onset is gradual, and perhaps the defect is only perceived accidentally or when the other eye becomes affected. In these cases it is usually the inferior segment which is the seat of the detachment.

Tension is usually normal at the time of onset, but during the first weeks or months cyclitis may develop, with hypotony, and in the later stages, perhaps after years, glaucoma may complicate the case.

If the case is seen a few days after the occurrence of the detachment, two kinds of change may be detected in the vitreous. The 
first is due to detachment of the vitreous, and consists in a certain deformation of the details of papilla, etc., which varies with movements of the eye as by an irregular parallactic movement, such as results from irregular astigmatism in lens or cornea. In the periphery of the vitreous, especially at the site of the detachment, we may find a diffuse opacity of the vitreous, as well as dark spots and streaks.

The detachment occupies a peripheral section of the fundus, with or without involvement of the macular area, but practically never the macula without the periphery. It occurs twice as often in the upper as in the lower part of the fundus, and, of course, corresponds with the defect in the field of vision. The most prominent form is that situated in the upper region, which looks like a large fluctuant sac hanging in the vitreous. At the other extreme is the form often seen in the lower region, which takes the form of a low elevation with practically no folds. Idiopathic detachment is distinguished as a rule from that due to tumour by a multiplicity of folds, and, when it takes the form of a rounded sac, its silky surface, its relative transparency and its mobility.

Ruptures of the retina, single or multiple, are almost always found in recent cases. They occur at the period of onset. The rupture sometimes takes the form of a crescent with its convexity towards the disc, the flap of tissue between the horns of the crescent being drawn forward into the vitreous like the flap of a valve. In other cases the perforation is of a punched-out character, and in such a case the detached portion may be seen lying in the vitreous in front of the rupture. In other cases there is a linear rupture at the ora serrata, no flap being seen. Whatever may be the type of rupture, it indicates the point of origin of the detachment. In a fresh case it often occupies the summit of the detachment, but at a later stage it may be found elsewhere, for example, in the upper part of the fundus after the detachment has shifted to the lower. The author states that the rule may be laid down, that cases of sudden onset have for point of origin a superior section of the retina, and present a crescentic or punched-out rupture, while cases of slow development are often in the lower part of the fundus, and, if they present a rupture, it is often at the ora serrata.

Small haemorrhages are sometimes seen scattered over the detached retina, but even more frequent are pigment deposits. Foci of choroiditis may be seen, either through the detached retina or through the rupture, and the frequency with which there is exact correspondence between the position of the rupture and that of a choroiditic focus shows that this association is not accidental.

The tendency of detachment of the upper region of the retina to shift to the inferior region of the fundus makes this form more 
resistent to treatment than that in which the lesion is from its onset situated in the lower area. In settling down, the detachment tends to invade the lateral regions, either on the nasal side, where it may extend as far as the margin of the disc, or on the temporal side, endangering the macula. On the manner in which this critical period is passed depends the question of the general diffusion of the detachment, or its reapplication, more or less complete and durable, in the upper region, and its definite localization in the lower. Having once reached the latter situation it tends to remain stationary. Even when a detachment reaches the very border of the macula, it is still compatible with good central vision.

Theories of pathogenesis. The three theories which have received most support are : the distension theory, the exudation theory and the traction theory.

The distension theory is based on the idea that if an eyeball is stretched or distended the less distensible retina comes to occupy the chord of the arc between its points of attachment. Such a distension is supposed to occur typically in a case of high myopia. The theory fails to explain why, in myopia, the detachment does not occur at the age when the myopia is progressive, but at a time when the elongation of the eye has long come to a standstill. Again, the idea that the retina is not extensible is not only insufficient, but is contrary to the facts.

The theory that detachment is caused by the forward pressure of a subretinal exudate fails on various grounds. Choroidal exudates do not form with sufficient rapidity to produce the sudden variety of detachment, they do not occur more frequently in the upper region of the fundus than elsewhere, and rather than go on distending one part of the retina to the point of producing a rupture such an exudate would tend to separate the retina over a larger area. Again, a sudden or rapid exudation sufficient to produce a large detachment would be sure to lead to an elevation of intraocular pressure. Another objection is that it is quite unusual for detachment of the retina to follow an acute choroido-retinitis. Sub-retinal exudates are regularly followed by reapplication of the elevated retina and the formation of cicatrices between retina and choroid. In the detachment associated with albuminuria rapid cure is as common as it is rare in idiopathic detachment. This theory must be reserved for the cases of detachment of slow development, occupying in a diffuse manner a large part of the fundus, without fluctuations of the surface and with a minimum of folds, with no discoverable rupture, and tending, after a short period to disappear spontaneously.

The traction theory was put forward by Leber in 1882, and was based on the cases of intraocular disturbance due to metallic foreign 
bodies, in which contraction of the vitreous led to detachment with rupture of the retina. Leber held that the ruptures so frequently observed in spontaneous detachment also resulted from traction exercised on the retina by the vitreous, and that the detachment was brought about by the passage of vitreous through the opening thus formed. Thus were to be explained the sudden onset and the absence of elevation of tension. The large retinal sac did not imply the formation of a sub-retinal exudate, but a mere displacement of the intraocular fluid. Gonin, at first an opponent of the theory, is now its most convinced advocate. The initial occurrence of a rupture by traction on the retina explains the sudden onset in a way which is impossible to the other theories. Again, the communication between the vitreous chamber and the subretinal space explains the sudden or rapid changes which may occur in the position or volume of a detachment, for example, the apparent cures and disappointing relapses which may occur during treatment. The persistence of the rupture is the cause of the persistence of many detachments, and, far from being a feature favourable to repair, as believed by von Graefe, it is its presence that justifies and explains the peculiarly hopeless prognosis of idiopathic detachment. The traction theory, however, involves certain important assumptions : the pre-existence in the vitreous of changes which will allow a displacement of the retina at the moment of the rupture, the accumulation of liquid in quantity sufficient to penetrate through the opening, and a cause of traction so energetic as to provoke the tearing of an opening in the retina.

The author believes that these assumptions are justified on both clinical and pathological grounds. The clinical picture of the early cases, as already described, makes it probable that there is a condensation of the vitreous body, shrinkage of its volume, and detachment of its posterior segment, and accumulation of fluid between it and the anterior surface of the retina.

After a series of brief answers to various objections which have been raised against the traction theory, the author controverts the idea that the downward displacement of a detachment indicates that the sub-retinal fluid is denser than that in the vitreous chamber, and explains in some detail what he conceives to be the probable mechanism of this change.

In his third chapter he proceeds to gather the evidence in favour of the traction theory which is derived from dissection of affected eyes, laying some stress on the value of macroscopic examination, which should not be entirely overlooked in favour of microscopic examination. After a general statement of the changes which one may find in the retina itself, in the vitreous, the uvea, etc., he proceeds to a detailed account of the conditions found in twelve cases already published, and in eight unpublished cases which he 
is now able to put on record. - These twenty cases, with four others which bear a certain resemblance to them, are tabulated in such a way as to show the age of the patient, the nature of complications, if any, the type of the detachment, the presence or absence of rupture of the retina, the condition of the intraocular fluids, and the state of the uvea. Two facts of importance which emerge are : the presence of one or more ruptures of the retina in every case where they were carefully looked for, and the identity of the pre-retinal and retro-retinal fluids; both facts which support the theory that it is attraction of the retina by a force acting on its anterior surface which is the factor common to all the cases of idiopathic detachment.

In Chapter IV, in which the author discusses the pathogenesis of detachment of the retina, he draws attention at the outset to the interesting fact that he is now in the unusual position of having to defend the traction theory against the objections of its principal author, Leber. In 1908, at Heidelberg, Leber, while adhering to the general principle of traction, adopted the idea that the force in question results; not from contraction of the vitreous, but from the contraction of a new-formed membrane on the anterior surface of the retina. The author, having reviewed the available pathological material and compared it with Leber's descriptions and illustrations, finds that in all material details he and Leber agree, but that the interpretation which the latter gives to the objective conditions rests on a too exclusive reference to the microscopic, and too little attention to the macroscopic appearances. Gonin admits the frequent presence of a new-formed membrane spread out on the anterior surface of the retina, but he easily disposes of the idea that the contraction of such a tissue is able to produce a retinal detachment with the characteristics belonging to the group under discussion. His pathological investigations have convinced him that idiopathic detachment is brought about by contraction of the vitreous, and that this is not caused by fibrillary degeneration of the vitreous itself, as first suggested by Leber, but by the presence in the vitreous of strands of epithelial cells undergoing a connective tissue transformation. The source of these cells is the epithelium of the ciliary body, and the cause of their proliferation and migration into the vitreous is to be found in nutritional disturbances in the anterior part of the uvea, while degeneration of the vitreous itself leads to the formation of lacunae which provide paths for the immigration of these cellular elements from the ciliary epithelium.

It is noteworthy that the conditions most favourable to the occurrence of idiopathic detachment of the retina-myopia and other forms of ectasia of the globe, senility, the presence of foci of choroiditis (syphilitic, tuberculous, etc.), are the very conditions 
which favour the occurrence both of circulatory disturbances in the anterior uvea and degeneration of the vitreous.

It is easy to understand the part played by trauma in the causation of detachment in eyes predisposed to it. A blow or jar, causing a sudden displacement of the intraocular media, may, by aggravating an already latent traction of the vitreous on the retina, cause a tear in the latter, and thus determine the onset of a detachment. This explains also the seriousness of loss of vitreous in a myopic or ectatic eye, or in one affected with choroiditis.

The concluding section of Gonin's report deals with detachments in hydrophthalmos, in vitreous haemorrhage, in metastatic ophthalmitis, in acute inflammations of the choroid and sclerotic, in albuminuric retinitis, exudative retinitis, etc. In the first of these the mechanism is the same as that applicable to idiopathic detachment in myopia. In vitreous haemorrhage traction is the cause of the detachment, but it is the late effect of the contraction of organized blood clot in the vitreous. In the other cases the formation of a sub-retinal exudate is the cause of the elevation of the retina.

A. J. Ballantyne.

\section{II-POST-HAEMORRHAGIC AMAUROSIS}

\section{(I) Terrien, Dr. F.-Post-haemorrhagic amaurosis. (Amaurose} hémorragique.) Arch. d'Ophtal., May, 1921.

(1) Terrien, in the paper here noticed, gives notes of two cases of defective sight following severe haemorrhage. The first case was that of a woman of 30 who had been confined four months previously. Haemorrhage had been very profuse, necessitating repeated injections of serum. She consulted Terrien four months later on account of defective vision. Ophthalmoscopic examination showed partial optic atrophy. Vision of right eye was $1 / 4$ and that of left $1 / 5$. Visual fields were generally contracted and the lower part of each field was nearly lost.

The second patient was a soldier, 24 years of age, who had been severely wounded in the left leg by a shell and not brought in for four days. Gas gangrene had set in and immediate amputation had to be performed. As he began to improve he complained of loss of sight. When Terrien saw him, about a fortnight after the injury, vision in right eye was fingers at $1.5 \mathrm{~m}$., and in left eye hand movements at a few centimetres. The pupils were moderately dilated and only reacted slightly to the brightest light though the convergence reflex was preserved. There was some haze of the 
optic discs and their margins were indistinct. The fields of vision were greatly contracted and, as in the previous case, the inferior part was almost abolished. The pupillary reactions recovered and there was a small improvement in vision (R.V. $1 / 10$; L.V. fingers at $25 \mathrm{~cm}$.).

In commenting on these cases Terrien admits the difficulties in the way of a pathological explanation. If they were due directly to the anaemia they should occur immediately; but this is hardly ever the case. He considers that the presence of slight symptoms of neuritis and the usual affection of the lower part of the field of vision give a clue to the explanation. His explanation is that the affection starts with retinal anaemia, which allows any toxin present to act more powerfully as it is less diluted, and that the superior part of the retina from the position of the head has a poorer blood supply. He points out that the rarity of such cases in purely traumatic cases, as contrasted with their greater frequency in diseased conditions, in which toxins would naturally be present, lend support to his theory.

E. E. H.

(2) Magitot.-The retinal vascular pressure in a case of posthaemorrhagic blindness. (La pression vasculaire rétinienne dans un cas de cécité post-hémorragique.) Ann. d'Ocul., Vol. CLVI, November, 1919.

(2) Magitot investigated a case of post-haemorrhagic blindness by the method of Bailliart, using, he says, a large enough ophthalmoscopic image to show the slightest or earliest pulsations. He gives a detailed account of the case, a woman of 52 , who was suddenly seized in the street with haematemesis, became unconscious, and had to be taken home. That afternoon there was much melaena, but she so far recovered as to be able to write two letters, showing that up to that time there was no gross visual upset. Next morning she had another syncopal attack with loss of consciousness and was taken to hospital, where some hours later she came to herself and stated that she did not see or hear any more. Hearing returned next day, but sight did not return till the sixth day, and then only in the left eye. Magitot was called in to see the case on the eighth day, and on the ninth day he found the general blood pressure by the Vaquez-Lauby apparatus 5 to 9.5 , while the Schiötz tonometer gave $10 \mathrm{~mm}$. Hg. for each eye. Right vision was 1 , with much contracted field; left hand movements. On the 26th day both discs very pale, especially left. The slightest touch on the eye caused the veins to empty themselves. Tension $15 \mathrm{~mm} . \mathrm{Hg}$. On the 36th day no spontaneous venous pulsation; tension $20 \mathrm{~mm}$. $\mathrm{Hg}$. The last observation was made on the 41st day and showed right field to be restricted to the central area and the upper outer quadrant, while the left had hand movements only 
in the temporal field. The arterial pressure (Vaquez) was from 5 to $10 \mathrm{~cm}$., retinal pressure 15 to 50 early in the case and later 30 to 65 .

Detailed figures are given and then Magitot makes the following observations :-

(a) Relation between the retinal and the general blood pressure. The general pressure after having remained to the 16 th day at $5 / 10 \mathrm{~cm}$. had gone up slightly by the 36 th day, and even on the 39 th had a tendency to progress $6 / 11.5$. During this lapse of time the graph shows the minimum retinal arterial pressure very low at first, rising rapidly to reach the normal on the 26th day. Thus there seems to be a real dissociation between the two pressures. The optic atrophy had progressed between the 9th and the 26th day. This anatomical fact might perhaps explain the rise of arterial pressure in the central artery, but Magitot puts this suggestion forward tentatively, for, as Bailliart has said, we do not yet know the formule of optic atrophy, which at first may not be the same for all the atrophies. Later when the arteries are filiform the vascular pressure is perhaps the same for all. Early in the case the blood pressure of a post-neuritic atrophy may differ from that of tabes or that after haemorrhage.

(b) Relation between venous pressure and ocular tension. The venous pulsations being almost always spontaneous, one may, therefore, conclude that the minimum pressure was very near the intraocular tension. The graph shows that the only time these pulsations were not spontaneous one induced their appearance with a weight so slight that one could hardly attribute more than $5 \mathrm{~mm}$. $\mathrm{Hg}$. between the minimum venous pressure and the ocular tension.

(c) Minimum and maximum of retinal veins. The figures between venous diastole and systole have always been close. The veins pulsating spontaneously one may deduce therefrom that the ocular tension would equalise the blood pressure. On the other hand, the slightest pressure on the globe flattened the central venous trunk, a phenomenon very obvious against the pale disc, but not seen in the branches.

(d) Pathogeny of post-haemorrhagic atrophy. The ninth day from the accident, when an ophthalmoscopic examination was made, she was examined sitting up and lying down, and a marked difference was noted in the figures of the blood pressure, the lowest figure being the vertical one, while the level of the diastolic arterial pressure was then so low as to border on that of the ocular tension ; in other words, the blood column seemed to have scarcely enough force to reach the eyeball. There was in the case first syncope, then blindness and deafness. Consciousness came back first, then hearing, and last of all and only partially, sight. Magitot traces this to the calibre of the different trunks to these 
various organs, while the auricular artery is closer to the basilar than the central artery is to the carotid. Thus, as in quinine poisoning, the blindness and optic atrophy here are due to the ischaemia of the third retinal neuron, the ganglion cells, whose death or destruction entails that of their axis cylinders, which form the optic fibres.

(e) The present observation leads to therapeutic conclusions. If the head had been kept very low at the start irreparable anatomical lesions of the nerves might have been avoided, while Magitot suggests the possibility of the Trendelenburg position being helpful, with injections of serum or of adrenalin to help the vaso-motor mechanism and raise the blood pressure.

W. C. Souter.

\section{III-PAPILLOMA OF THE CORNEA}

Garraghan, Edward F., A.M., M.D. (Chicago, Illinois).Papilloma of the cornea. Amer. Jl. of Ophthal., Vol. IV, No. 10. October 1921.

Garraghan publishes a note on a case of papillomatous growth of the cornea, the rarity of tumours of this kind and their tendency to recur lending special interest to the case.

The macroscopic appearance was that of an extensive granular tumour, like a raspberry, which was firmly attached to the corneoscleral margin, and lying rather freely upon the eyeball. Only a small section of the iris and pupil was visible, and a probe could be passed beneath the tumour all round the cornea and conjunctiva, except at the region of several blood-vessels which passed from the centre of the tumour through the substance of the conjunctiva to the inner angle of the eye. The tumour bled easily on manipulation.

Microscopic examination: the pathological report was to the effect that the tumour was a papilloma with mild secondary inflammatory reaction.

The tumour was removed, and the cornea began to clear, the conjunctiva of the bulb being entirely free of the tumour. In two weeks, however, there was recurrence over the cornea, and two large vessels were seen to pass from the tumour into the conjunctiva. These vessels were ligatured and the tumour cauterized as at the first operation. Slight recurrence took place on several later occasions, and cauterization was employed, the last occasion being fairly recent, so that complete cure cannot be said to have been achieved. 
The author gives a short historical survey of epibulbar tumours, the majority of which originate in the corneo-scleral margin, or conjunctiva, and spread until they involve the cornea.

The tendency of these tumours is to become malignant, a feature which should always be borne in mind. A coloured plate gives a good illustration of the macroscopic appearances before operation.

J. Hamilton McIlroy.

\section{IV-NON-SURGICAL TREATMENT OF MALIGNANT EPIBULBAR NEOPLASMS}

Heckel, Edward B. (Pittsburg). - Non-surgical treatment of malignant epibulbar neoplasms. Arch. of Ophth., March, 1922.

Heckel reports two cases of malignant epibulbar neoplasms cured by X-rays. The material facts are as follows:-

Patient, aged 71 years, whose right eye had been affected for about one year. A small piece of the neoplasm was examined microscopically and the portion nearest the cornea contained several outgrowths with well-developed epithelial pearls. The diagnosis of epidermoid carcinoma was made. Seven exposures to X-rays extending over 35 days were made. In the result complete cure was obtained.

A patient, aged 61 years, whose right eye had been affected for about six months. The neoplasm was found to be a squamous-celled carcinoma. The case was exposed to X-rays on twelve occasions, spread over a period of seventy days. The result was successful.

Heckel remarks that the principal commandment of the cancer decalogue is, "do not cut across a cancer and leave a part behind. The part remaining will grow more rapidly than if it had been let alone altogether." As regards technique, the face is covered with a piece of tinfoil, perforated with a small hole a little larger than the neoplasm, and so placed that when the patient is told to look in a certain direction the hole is directly over the neoplasm.

Where the neoplasm projects through the lid aperture as a cauliflower growth, it is well to excise the protruding mass and then to follow this immediately with the Röntgen ray or radium.

S. S. 


\section{V-REMEDIES}

(1) Zimmermann, Charles (Milwaukee, Wisconsin). - Parenteral injections of milk in diseases of the eye. Amer. Jl. of Ophthal., January, 1921, p. 18.

(1) Zimmermann gives a short survey of the therapeutics of milk injections, with a critical review of the results of various workers, and a report of two cases of his own.

He describes the technique and the general reaction of parenteral injections, but points out that the mode of action is not yet sufficiently known. High temperature appears to have a helpful influence upon the course of localised affections, in support of which view the author instances the improvement of trachoma in an epidemic of scarlet fever among 40 children. Whether this is due to the high temperature acting deleteriously upon the microorganisms, or to leucocytosis, or to hyperaemia and transudation at the site of local inflammation is a matter of theory at present.

Zimmermann quotes favourable results from milk injections on the part of Berneaud of Kiel, Maschler, Jickeli, etc., and doubtful or negative results on the part of many others.

His own cases (phlyctenular kerato-conjunctivitis with pronounced strumous diathesis) showed very gratifying results from single injections. He sterilized the milk for 10 minutes previous to injection into the gluteal region.

\section{J. Hamilton McIlroy.}

(2) Bussy, L., and Japiot, P. (Lyon).-Radiotherapy in interstitial keratitis. (La radiothérapie dans la kératite interstitielle.) La Clin. Ophtal., February, 1921.

(2) In a very moderately worded article Bussy and Japiot relate their experience with very small doses of X-rays in the treatment of interstitial keratitis. The following is a summary of the paper:- $\mathrm{Up}$ till now radiotherapy has not been systemfatically tried in interstitial keratitis. Yet this is a disease in which it is specially indicated because it is chronic, because it is characterized, in the beginning, by infiltration between the corneal lamellae of young connective tissue cells which are radio-sensitive, and that without severe lesion of the corneal tissue. Experience justified the above hypothesis. To be efficacious the treatment must be begun during the period of infiltration, before cicatricial changes have been caused. The younger the patient, and the more the disease is of the inflammatory type, the more favourable are the results. The hereditary syphilitic forms are the most amenable to the treatment. In strumous children the results are less favourable. The method employed by the authors is to depend 
on what they themselves describe as homoeopathic doses. Such doses, where only penetrating rays are used, do not necessitate terrifying apparatus to protect the skin and parts of the eye not under treatment, such as would otherwise be necessary in young children whose growing cells are very radio-sensitive. The authors apply to the closed eye, without special localisation or protection, very small doses of penetrating rays during five séances of five minutes each, the séances being separated by a week. It is probable that these radiations act by mobilizing rather than by destroying the cells. The method should be used in conjuriction with the ordinary methods, not as a substitute for these. "It causes a very rapid quietening of subjective phenomena, such as pain, photophobia and blepharospasm. It may considerably shorten the duration of the disease and that, especially, at the expense of the stage of vascularization. Finally, if employed early, it leads to an absorption of the infiltrates and clearing of the cornea in a manner which does not seem to occur with any other method."

ERNEST 'ThOMSON.

\section{BOOK NOTICES}

Ophthalmological Society of Egypt. Bulletin of 1921. Cairo: 1921.

This volume of 79 pages includes the proceedings of the eighteenth annual meeting held on March 4, 1921, in the Pathological Museum of the Kasr el Ainy Medical School. The first 40 pages of the report are occupied with the discussion on the prophylaxis of trachoma and the various forms of ophthalmia, a sequel to the appointing of the Committee in March, 1920, to study and report on the subject. This discussion has been touched upon this year in these columns (page 126). Since that note was written S. C. Hebard, Senior Inspector of the Ministry of Education, has protested against the report of the Committee. $\mathrm{He}$ points out that the Committee has not taken steps to ascertain the medical work actually done in Government Schools, and recommends methods of medical inspection which were in operation long before the Ophthalmological Society of Egypt existed. In point of fact, the Government Schools have been medically inspected since 1864. Hebard objects also to the Committee's recommendation that the Ministry should be advised to appoint an adequate number of medical officers who have previously had a short training in ophthalmology. With one exception all the doctors in the service of the Ministry have been trained in ophthalmology, 